\title{
A Week in the Life of Three Large Wireless Community Networks
}

\author{
Leonardo Maccari ${ }^{\mathrm{a}}$, Renato Lo Cigno ${ }^{\mathrm{a}, *}$
}

${ }^{a}$ DISI, University of Trento

\begin{abstract}
Wireless Community Networks (WCNs) are created and managed by a local community with the goal of sharing Internet connections and offering local services. This paper analyses the data collected on three large WCNs, ranging from 131 to 226 nodes, and used daily by thousands of people. We first analyse the topologies to get insights in the fundamental properties, next we concentrate on two crucial aspects: $i$ ) the routing layer, and $i i$ ) metrics on the centrality of nodes and the network robustness. All the networks use the Optimized Link State Routing (OLSR) protocol extended with the Expected Transmission Count (ETX) metric. We analyse the quality of the routes and two different techniques to select the Multi-Point Relay (MPR) nodes. The centrality and robustness analysis shows that, in spite of being fully decentralized networks, an adversary that can control a small fraction of carefully chosen nodes can intercept up to $90 \%$ of the traffic. The collected data-sets are available as Open Data, so that they can be easily accessed by any interested researcher, and new studies on different topics can be performed. WCNs are just an example of large wireless mesh networks, so our methodology can be applied to any other large mesh network, including commercial ISP networks.
\end{abstract}

Keywords: mesh networks, community networks, wireless ad-hoc networks, network topology, privacy, network analysis, centrality metrics, robustness analysis.

\section{Introduction}

A Wireless Community Network (WCN) is a wireless mesh network created by a local group of users to have an alternative, self-managed, community-based networking infrastructure. A WCN serves two purposes: It allows inter-user interactions (messaging, talking, sharing etc.), and it brings Internet connectivity where it is not present. WCNs are flourishing. Many European cities feature WCNs with hundreds of nodes: in Athens a single WCN includes more than 2,400 nodes, while in Spain, the Guifi network is a composition of WCNs that counts more than 23,000 nodes and growing. Thousands of nodes connecting tens of thousands of individuals, families, associations, public offices with a non-profit approach and a community-based organization. After an initial interest in their early stages [1], WCNs have lately re-attracted the attention of academia and research funding $[2,3]$, and they are becoming a strong asset in reducing the digital divide and pushing broadband networks from the bottom up.

The goal of this paper is to analyze the main features of three large European WCNs, with particular focus on routing aspects and on centrality and robustness metrics.

\footnotetext{
This work is partially funded by The Trentino programme of research, training and mobility of post-doctoral researchers, incoming Post-docs 2010, CALL 1, PCOFUND-GA-2008-226070, and by the European Commission under Grant Agreement No. FP7-288535 "CONFINE”: Open Call 1, Open Source P2P Streaming for Community Networks -OSPS-

${ }^{*}$ Corresponding Author

Email addresses: leonardo.maccari@unitn.it (Leonardo Maccari), renato. locigno@unitn.it (Renato Lo Cigno)
}

\subsection{Contribution}

This paper extends the initial findings on a small portion of the data presented in [4], leveraging the analysis, the metrics and theoretic contributions published in [5, 6]. It offers an original combination of insights not present in the existent literature. First of all, three different large networks are monitored for an entire week, exploring their stability and different characteristics and finally providing a novel comparative analysis of the three networks.

Second, WCNs do not strictly focus on Internet connectivity, as the large commercial networks analyzed in the literature. Instead, the participants of a WCN perceive the network as an alternative communication media that offers a higher degree of privacy and neutrality. For this reason they try to use the internal services of the network as an alternative to external commercial services. In the light of the recent world-wide discussions on privacy, neutrality and forced disconnections, WCNs represent successful networks based on a somehow revolutionary societal approach. For this reason it is particularly important to study their development, describe their features and verify how much they match the expectations, raised even by mainstream media $^{1}$. One of the contributions of this paper is the analysis of the robustness and of the centrality metrics of WCNs, that give an unbiased overview of how much these expectations are reflected in real networks.

Third, we focus on specific issues that have been ignored by previous works, as the analysis on the choice of Multi-Point

\footnotetext{
${ }^{1}$ See, for instance, recent coverage from the New York Times "U.S. Promotes Network to Foil Digital Spying" http://nyti.ms/1r6yltT
} 
Relays (MPRs) in the Optimized Link State Routing (OLSR) protocol. MPRs are key nodes used in the OLSR protocol that have been largely debated in the literature, most of the times using a theoretical or simulative approach. We believe this is the first attempt to evaluate on real topologies how MPRs could impact the performance not only in terms of signalling, but also in terms of accuracy in finding the best routes.

Finally, and contrarily to the majority of the works in literature, we release all the data we have collected and the software we developed to encourage more researchers to investigate on this topic, so that new comparative research can be based on this work. We will continue to monitor the three networks and, if possible, to extend the monitoring to new ones and enrich the public data-set with new features ${ }^{2}$.

\subsection{Related Work}

Several works describe the features of wireless mesh networks. In some cases, detailed analysis were made on small wireless networks [7, 8], in some other cases large networks providing Internet access were analyzed [9, 10, 11]. Nevertheless, there is a great difference between a commercial access network and a large WCN, which offers some unique challenges [12] and displays some unique features.

Recently the topological properties of Guifi have been studied [13], and in a previous work [4] we analysed some feature of the Ninux network.

This paper goes beyond the state of the art and focuses on some currently unexplored specific issues. Among these, we will study the centrality metrics applied to WCNs, and, specifically, group centrality metrics. These are metrics that have been largely used in social science, but have been applied to wireless networks only recently $[14,15]$, but never to networks as large as the ones we consider.

Finally, many works in the literature address the problem of finding the optimal MPR set for a network [16, 17, 18, 19]. Most of these works are based on geometric evaluations or simulations and, to the best of our knowledge, there is none estimating the impact of different MPR choice strategies in real large topologies as we do in this work.

\section{Overview of the Networks and the Measurements}

The three networks we consider are Funk Feuer Wien and Funk Feuer Graz in Austria, and Ninux in Italy: FFWien, FFGraz, and NNX for short. They have different management structures and "philosophy", but they all exploit the OLSR routing protocol to maintain the network topology and compute routing.

\subsection{Nodes' Configuration}

The majority of the nodes use either one of two solutions: $i$ ) boxed indoor equipment, or $i$ ) commercial devices for outdoor use.

\footnotetext{
${ }^{2}$ The software developed and data-sets collected for this work are available at http://disi.unitn.it/maccari/CN
}

In the first case devices such as the TP-Link TL-wr841nd ${ }^{3}$ are modified using outdoor antennas, powered over Ethernet and enclosed in a plastic box. This is a low cost solution, easy to deploy since it relies on omnidirectional antennas that do not need to be aligned. The drawbacks are short ranges, higher interference, and a lower throughput.

In the second case devices such as the Ubiquiti nanostation ${ }^{4}$ are used. They have embedded panel antennas with a beamwidth of 40 degrees or parabolic antennas with a beam-width of 10 degrees. This second solution needs more expertise to be installed, but guarantees longer ranges and higher bit rates. Using directional antennas, it is often necessary to install more than one device to connect to neighbor nodes. Each device is connected to the others via Ethernet; this configuration is called a super-node. A super-node implements cross-AP routing and maintains a large horizontal 'virtual' coverage angle while featuring long ranges and high bit rates.

The communication technology used is a mixture of IEEE $802.11 \mathrm{~g} / \mathrm{a} / \mathrm{n}$ standards with preference for $802.11 \mathrm{n}$ to achieve higher bit rates and use the $5 \mathrm{GHz}$ frequency that is generally less crowded of consumer devices.

Each WCN or user, decides what is the best Operating System (OS) for the nodes, and the choice depends on many factors. As a general rule, using the OS shipped with the device has higher stability and better performance due to a better integration with the hardware. As a drawback it may not allow the users to modify the routing protocols or use the ad-hoc mode.

\subsection{The OLSR routing protocol}

Some comprehension of the OLSR protocol is needed to better understand the remaining of the paper. Since OLSR is well known and described in the literature [20], we give just a brief description of its principles. OLSR is a link state protocol based on Djikstra algorithm, where only bi-directional links are included in the link set, and they are discovered using a "hello" procedure that pairs beacons, called HELLO messages, broadcast by each and every node in the network.

Let's now introduce some specific notation. In a network $N$ each node $j$ has a set of one-hop neighbors $N_{1}(j)$ reachable with a direct link and discovered with the hello procedure. Nodes are included in $N_{1}(j)$ only if they have proper symmetric links. Every node $j$ also has a set of two-hop neighbors $N_{2}(j)$ reachable through some node $i \in N_{1}(j)$ with exactly two hops (by construction $\left.N_{1}(j) \cap N_{2}(j)=\emptyset\right)$.

$N_{1}(j)$ and $N_{2}(j)$ are built as follows: every node $j$ periodically sends an HELLO message to build the knowledge about the 1-hop neighborhood. Furthermore, HELLO messages contain the IP addresses of every node in $N_{1}(j)$. Through the HELLO messages of its neighbors, at steady state, $j$ has the full knowledge of $N_{1}(j)$ and $N_{2}(j)$.

Node $j$ elects, among the nodes in $N_{1}(j)$, a set of MPRs $M(j)$. $M(j)$ satisfies the following condition: every node in $N_{2}(j)$ must have at least a symmetric link towards a node in $M(j)$. Thus if

\footnotetext{
${ }^{3}$ See www.tp-link. com/en/support/download/?model=TL-WR841ND

${ }^{4}$ See www. ubnt. com/airmax
} 
$i \in M(j)$ it "covers" some of the nodes in $N_{2}(j): M(j)$ completely covers $N_{2}(j)$. When $j$ selects one MPR $i$ it informs $i$ that it has become one of is MPR selectors. Each MPR starts behaving as follows:

- It periodically generates Topology Control (TC) messages. A TC contains the list of the IP addresses of its MPR selectors;

- It rebroadcasts the TCs received from its selectors.

TC messages contain an approximation of the local topology around an MPR and are received by all the nodes, given the construction procedure of $N_{1}(j), N_{2}(j)$, and $M(j)$. In this way, each node has enough information to compute the shortest path route to any other node. Since only MPR nodes retransmit the TCs, TCs will reach all the nodes in the network using a fraction of re-transmissions compared to plain flooding. Minimizing the size of each $M(j)$ is thus important to minimize the union of all the $M(j)$, which in turn minimizes the number of generated and forwarded TC messages.

In the network under consideration OLSR is configured to use a quality metric on links: the Expected Transmission Count (ETX) metric. ETX estimates the average number of times a packet needs to be transmitted to reach a neighbor, taking into account losses due to collisions and interference. Since the timer used between every HELLO message is known, each node $j$ estimates the number $e$ of HELLO messages that it should receive from one neighbor $i$ in a given time window. Node $j$ counts the number $r$ of HELLO messages actually received from $i$ in the window and specifies in its HELLO messages the ratio $r / e$. The value $r / e$ is called the link quality $(L Q)$ of the link from $i$ to $j$. Node $i$ will do the same, so that node $j$ knows both the $L Q$ value and the reverse value (neighbor link quality, $N L Q$ ). Since any unicast transmission in 802.11 requires a data frame and an ACK in the opposite direction, the probability of successfully sending a packet is approximated by $L Q \times N L Q$. The average number of frames needed to successfully send a packet is thus estimated as $E T X=\frac{1}{L Q \times N L Q}$. MPRs add to the TC messages the ETX for each of their selectors. If ETX is used, the minimum cost route is computed with Dijkstra's algorithm on a weighted graph. Since TC messages contain the ETX metric only for the links between an MPR and its selectors, $j$ has only an approximated knowledge of the network. In practice, MPRs hide the presence of some links.

The advantage of using MPRs to reduce overhead, as well as the utility of the ETX metric is hotly debated among OLSR users. Without entering the debate, we note that ETX is a very crude metric and it is estimated on short messages sent in broadcast, i.e., at the minimum physical transmission speed, which means that ETX is hardly representative of the actual quality of the unicast link between two nodes. It is instead meaningful to estimate the diffusion of routing signalling messages.

\subsection{The Data Gathering Process}

The data collection relies on the information exposed by the OLSRd daemon. For each community network we store snapshots of the topology as a weighted non-directed graph $G(\mathcal{N}, \mathcal{L})$ at regular time intervals. $\mathcal{N}$ is the set of all nodes, with supernodes counting as a single node, and $\mathcal{L}$ is the set of all links $l_{i j}$ as selected by OLSR. The weight $c_{l} \forall l \in \mathcal{L}$ is either 1 for minimum hop routing or the OLSR ETX metric measured on $l$. Let $P_{i, j}$ be the best path from node $i$ to node $j$ as selected by OLSR and

$$
w\left(P_{i, j}\right)=\sum_{l \in P_{i, j}} c_{l}
$$

be its cost or weight.

In all the networks, OLSR is configured to force each node to select all its neighbors as MPRs. As a result every node is an MPR and thus every node has a complete knowledge of the topology. To know the entire topology, it is sufficient to extract information from one node. The OLSRd software daemon can be configured to export the network topology, and all the networks publish this information. Appendix A describes in detail how the data is collected and what pre-processing is applied to it to make the topological description of the networks more meaningful.

\begin{tabular}{c|c|c|c} 
& FFGraz & FFWien & NNX \\
\hline first sample & $01 / 07 / 14$ & $01 / 07 / 14$ & $01 / 14 / 14$ \\
\hline last sample & $01 / 13 / 14$ & $01 / 13 / 14$ & $01 / 20 / 14$ \\
\hline samples per day & 144 & 288 & 288 \\
\hline samples time interval & $10 \mathrm{~min}$ & $5 \mathrm{~min}$ & $5 \mathrm{~min}$
\end{tabular}

Table 1: Summary of the data-sets collected

The data collection process is performed by a daemon written in the Python language that downloads new topologies in real time and saves them in an SQL database. As we already mentioned, the source code is published as Open Source, and the database as Open Data, so that any researcher wishing to use the data used for this paper is free to access it.

The data has been collected in a period of one week for each network, in Table 1 we report a summary of the data collected for each network. The number of samples of FFGraz is smaller due to the larger sampling period, so that for a one-day observation we have 288 samples for FFWien and NNX and 144 for FFGraz.

\section{Main features of the networks}

Table 2 shows the key features of the networks. We can note that the three networks are different, but share some topological characteristics. The NNX network is the smallest of the three, FFGraz is the one with the highest density (the density of a graph $G(\mathcal{N}, \mathcal{L})$ is defined as $\left.\delta=\frac{2\|\mathcal{L}\|}{\|\mathcal{N}\| *\|\mathcal{N}-1\|}\right)$. The density in a graph with constant connectivity degree decreases with the number of nodes, so even if NNX has a smaller average degree compared to FFWien, it has a higher density. The proportion between leaf nodes and non-leaf nodes is similar in the three networks. Another measure of interest is the average local clustering coefficient of the network $\left(C C_{1}\right)$, that expresses the 


\begin{tabular}{c|c|c|c} 
& FFGraz & FFWien & NNX \\
\hline av. number of nodes & 144 & 236 & 131 \\
\hline av. number of links & 207 & 421 & 153 \\
\hline density $\delta$ & 0.020 & 0.015 & 0.017 \\
\hline average degree & 2.88 & 3.63 & 2.35 \\
\hline leaf nodes & $63(43 \%)$ & $77(32 \%)$ & $51(38 \%)$ \\
\hline average ETX & 2.0 & 1.46 & 1.24 \\
\hline average $C C_{1}$ & 0.1934 & 0.2358 & 0.0347
\end{tabular}

Table 2: High level features of the networks under analysis

local density of links around a node. The local clustering coefficient of node $i$ with one-hop neighborhood size $n_{i}=\left|N_{1}(i)\right|$ is defined as:

$$
C C_{1}(i)=\frac{2\left|\left\{l_{k, j}: k, j \in N_{1}(i) \wedge l_{k, j} \in \mathcal{L}\right\}\right|}{n_{i}\left(n_{i}-1\right)}
$$

In average, Ninux has a much smaller $C C_{1}$ compared to Funk Feuer Graz and Funk Feuer Wien.

\subsection{Time Evolution of the Networks}

During the observation on the whole week we measured only a small fluctuation in the number of nodes and in the number of links, mainly due to a small subset of leaf nodes with instable links that periodically exit and enter the main connected component of the network. This is reported in Fig. 1 where one snapshot per hour for the full week is reported. Note that the fluctuations of leaf nodes/links (the upper plot) have a marginal impact on topology and routing.

Fig. 2 reports the persistence of each link of the networks, that is, the fraction of snapshots over the total for which the link has been present in the network. From this data we removed the links that have a persistence smaller than $1 \%$. This is to avoid to consider sporadic links generated by test devices or temporary changes in the nodes configurations. There is a difference among the networks, while NNX shows a very high persistence for the majority of the links, FFWien and FFGraz present more temporary links. Still, in both cases there is a large core of links that is present in all the snapshots.

The fluctuation of the number of nodes and links does not impact the macroscopic routing characteristics of the topology. The reason is that fluctuations normally happens in marginal nodes and links, so that the average length and cost of the shortest paths are not influenced. This observation is reported in Fig. 3, together with the average ETX, which doesn't vary significantly, either.

Fig. 4 reports the clustering coefficient of each network. Sect. 5 discusses how $C C_{1}$ directly influences the robustness of the networks.

Since the network features are stable, from now on we limit the analysis to data relative to one day of observation, in order to reduce the effects of fluctuations and simplify the interpretation of results. Since the full data-set is available on-line, all the results are reproducible also for other time-periods. We have observed some other days obtaining consistent results.
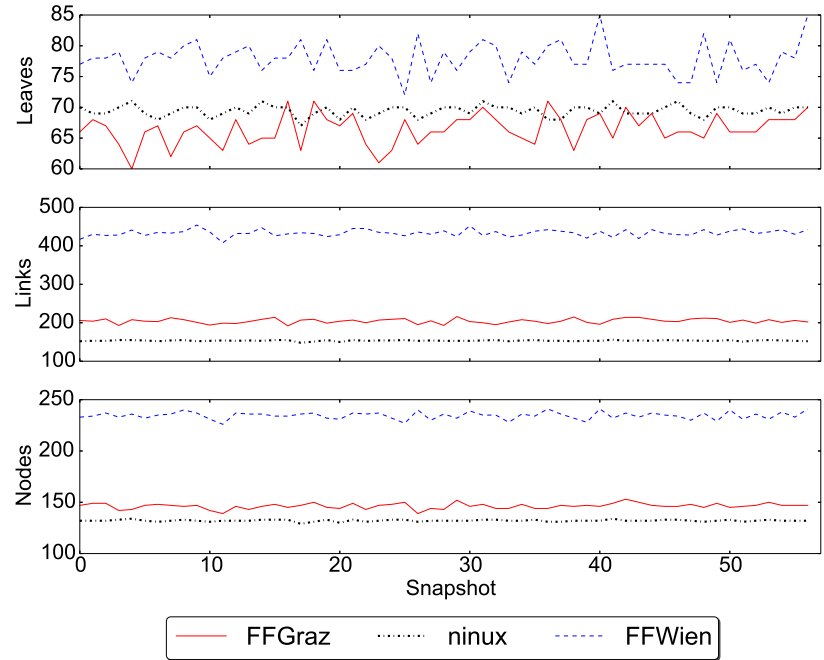

Figure 1: The number of nodes, leaves and edges for the three networks during the whole week.

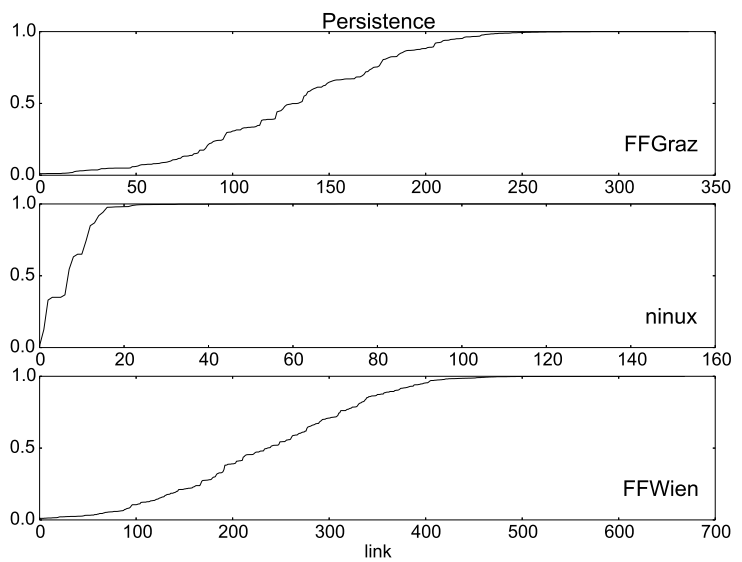

Figure 2: Persistence of the links for each network

\subsection{ETX and Degree Analysis}

Fig. 5 shows the average degree distribution of the three networks. For each node the degree is averaged over all the snapshots and rounded to the closest integer. The figure reports also the power law and exponential curves that best fit the points (using minimum mean square error). Since the number of samples and the dimension of the network are not large enough to compare it with existing literature on power-law networks, we do not indulge in the analysis of the degree distribution; we just observe that qualitatively the distributions are very skewed. This is not surprising also considering the results on another WCN presented in [13].

Fig. 6 shows the empirical CDF of ETX for all the links and all the snapshots. A large portion of the links have an ETX $\simeq 1$. Independently from the network the distributions have a long tail of higher values, but the slope is different. Fig. 7 reports the average ETX for every link computed on all the snapshots. In 


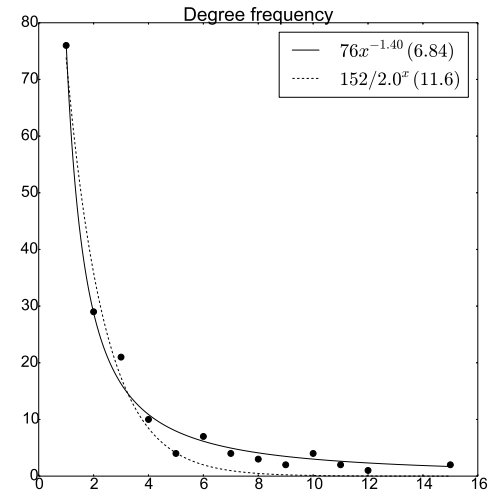

(a) Funk Feuer Graz

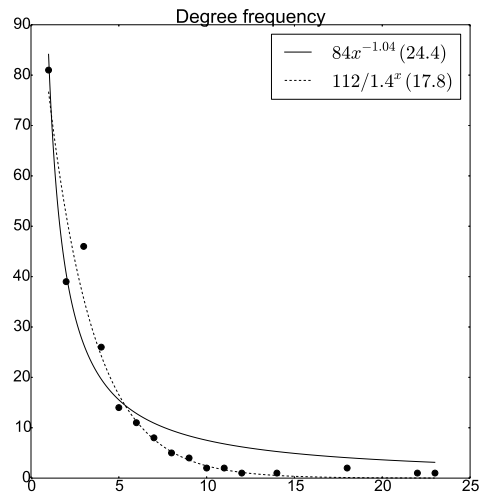

(b) Funk Feuer Wien

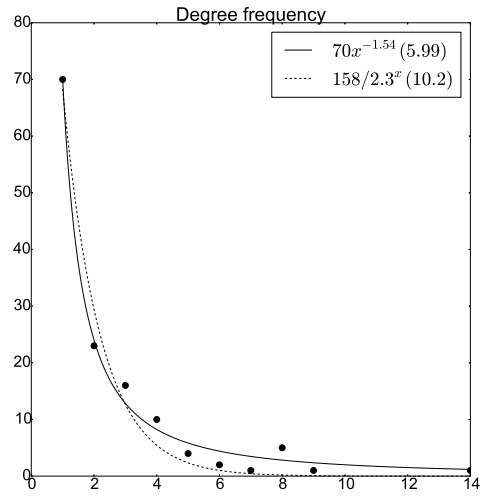

(c) Ninux

Figure 5: Degree distributions with exponential and power law fitting curves
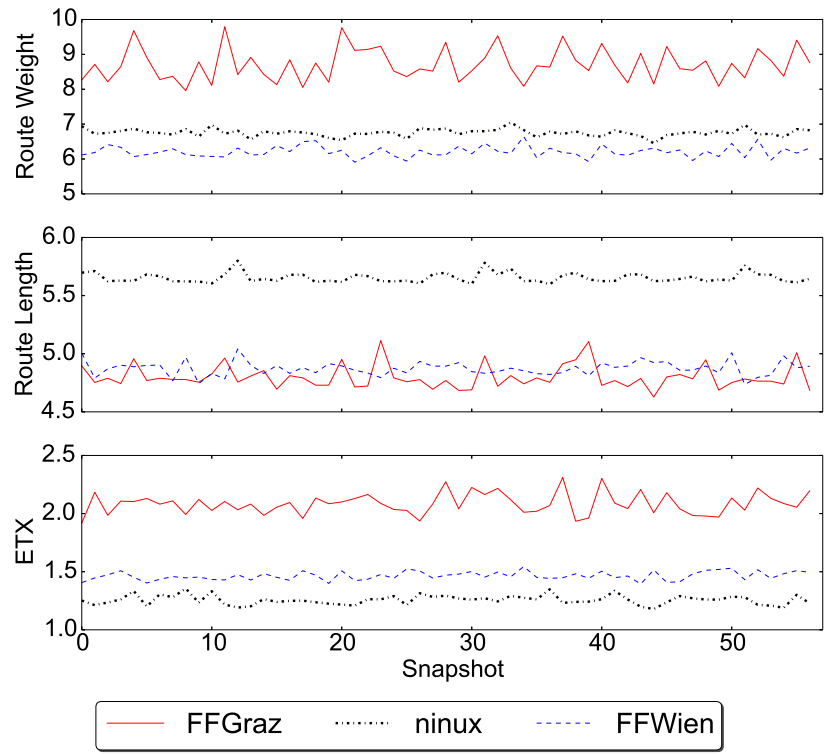

Figure 3: Average ETX value, route length, and route weight for the three networks during the whole week

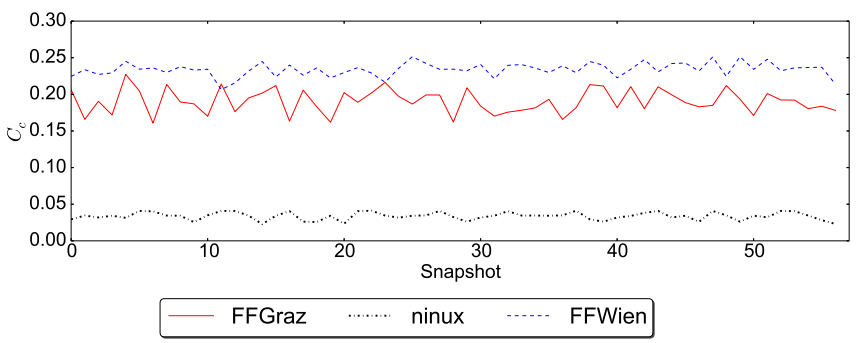

Figure 4: Clustering Coefficient for the three networks during the whole week

Ninux it has a smaller range compared to Funk Feuer networks. In all networks and all links ETX is relatively stable: the av-

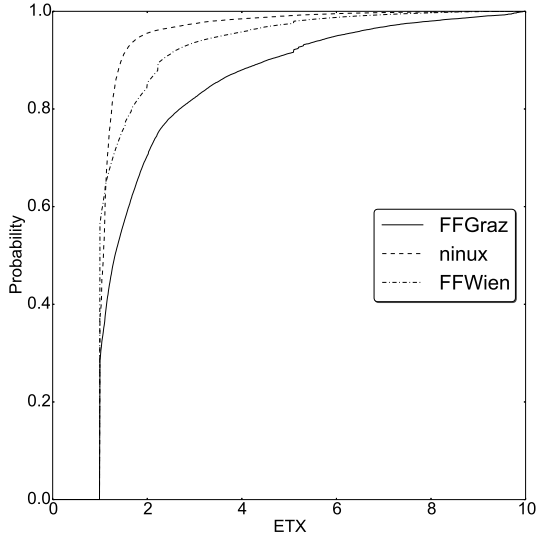

Figure 6: ECDF of the ETX value for the three networks

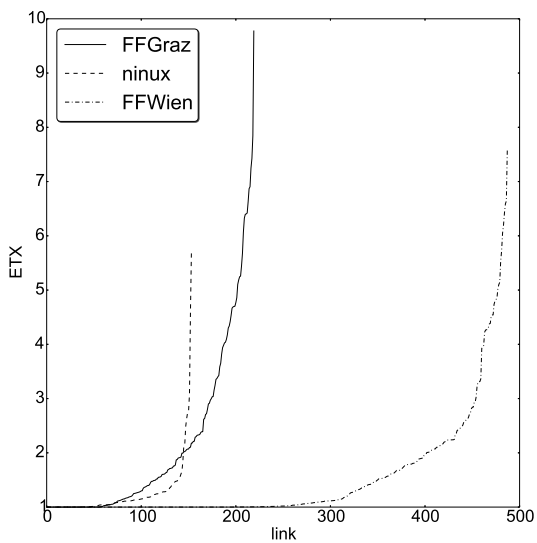

Figure 7: Average ETX of each link in the three networks (links are ranked by ETX)

erage standard deviation of the ETX on each link is less than $16 \%, 10 \%$ and $7 \%$ for Funk Feuer Graz, Funk Feuer Wien and 
Ninux respectively. This actually contradicts the common assumption that in WCNs the links are unstable and their quality is highly variable.

In the past, due to the unavailability and price of devices, the networks were set-up using omnidirectional antennas, and adhoc wireless mode. This allowed maximum flexibility, almost no planning, but it also yielded poor performance and high interference. Today, a large number of nodes are equipped with directional antennas, and the super-node configuration (see Appendix A) is used to enlarge the coverage angle of each node. In some cases the original firmware is not changed on the radio device, and master-client configurations are used. This requires some effort in network planning, but it also allows the use of proprietary extensions that further increase the performance of each link. In the case of Ninux we verified these assumptions directly with people from the WCNs that confirmed that the majority of the nodes are configured to have one link per each radio.

We note some differences. Ninux has the best average quality, and more than $90 \%$ of the links have ETX $\leq 2$, while Funk Feuer Graz has the highest average ETX and the highest standard deviation per link; it is the one with the highest density, suggesting that in Funk Feuer Graz it is more common to to use the same device for more than one link.

\section{The Routing Layer}

Once the weighted network graph is known, we can compute all the shortest paths from any couple of nodes in the network. Since every node is an MPR this is the same information that a node in the network uses to compute its routing table.

Fig. 8 reports the distribution of the paths' weight $w\left(P_{i, j}\right)$ computed both with a simple hop-count metric $\left(c_{l}=1 \quad \forall l \in \mathcal{L}\right)$ and considering the ETX metric as OLSR does. The graph is based on all the routes computed on all the snapshots of a oneday data-set. $w\left(P_{i, j}\right)$ is quantized to integer values for readability.

Funk Feuer networks have the mode at 5 hops, while Ninux despite being the smallest of the three has the mode at 6 hops (this can be explained observing that it is the network with the smallest average degree). The difference between the curves representing length and weight can be better appreciated looking at the cumulative distribution. In Funk Feuer Wien and Ninux the curves are very close to each other, while in Funk Feuer Graz the difference is much more evident. This shows that the highest quality of the links in Funk Feuer Wien and Ninux is directly reflected in the paths' weight $w\left(P_{i, j}\right)$ computed on ETX. Notice that Funk Feuer Graz features a very different distribution of $w\left(P_{i, j}\right)$ computed on ETX compared to the other networks. The reason lies in the particular topology that leads to some nodes with high centrality but bad links and will be more clear after the discussion in Sect. 5. Fig. 10 shows a snapshot of the Funk Feuer Graz topology where, albeit only with a trained eye, this somewhat pathologic situation can be seen.

Fig. 9 reports boxplots of $w\left(P_{i, j}\right) \forall i, j$ based on ETX grouped by number of hops, and computed in each network, for each

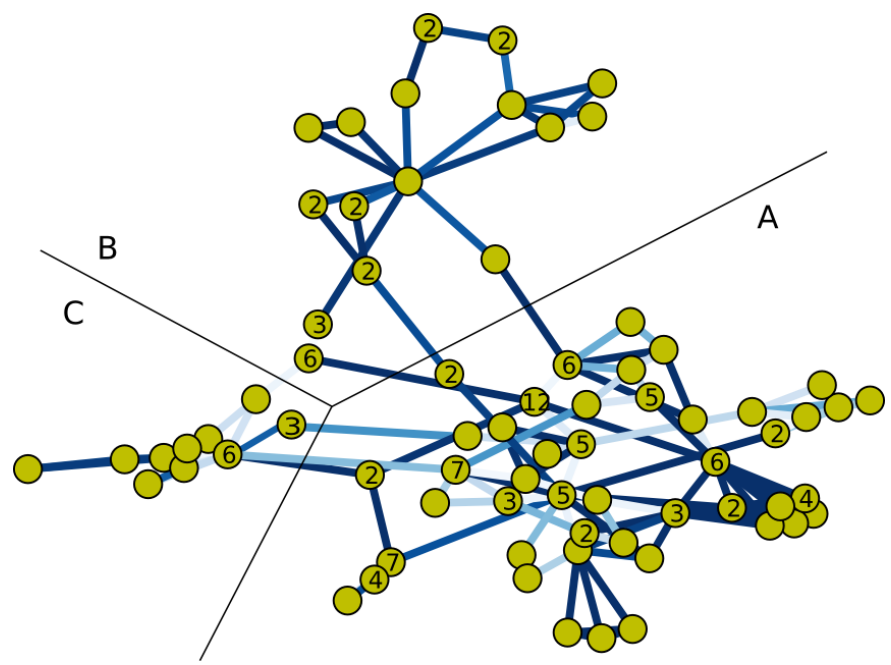

Figure 10: Compressed Funk Feuer Graz topology, the number in circles indicates the number of attached leaves. The density of the link color is proportional to its goodness (light blue $=$ bad link). 3 clusters can be identified, two of them connected with weak links.

snapshot. The boxplots show some intuitive but interesting results. For the FFWien and NNX networks the median of the weights (the red line in the box) is very close to the minimum value (the bottom whisker). Also, the first and third quartile (the boxes) are close to each other, which means that more than $75 \%$ of the routes of the same length have a very similar behaviour. In FFWien with routes longer than 6 hops the upper whisker starts diverging from the median, indicating a higher variance of $w\left(P_{i, j}\right)$. This is not evident in the Ninux network. In FFGraz there are two separate effects, the first one is that even for shorter paths the boxes are much wider than for the other networks, the second is that, due to the particular topology of the network, the variance of $w\left(P_{i, j}\right)$ is not monotonic.

As a concrete application of this analysis, imagine that a certain service is placed on a single node $i$ in the network, and must be reached by any other node $j$, for instance, a VoIP Border Media Gateway. The weight $w\left(P_{i, j}\right)$ influences the service quality since it is an estimate of the average number of wireless frames that need to be sent along the path for the packet delivery. In Ninux if $i$ is chosen to be at an average distance of 6 hops from any $j$ (the mode of the route length as seen in Fig. 8c) the perceived quality of the service will be similar for the large majority of nodes since the boxplots for length 6 is narrow. This is not true for FFGraz, for which, among the routes that have a length 5 (the mode for FFGraz) the distribution of the weights is much wider. Thus the perceived quality of the service will largely differ depending on the position of $j$. Since the average distance of $i$ from any node $j$ can not be arbitrarily reduced, the only solution to have a more homogeneous quality is, if possible, to use two servers, thus doubling the effort. Other similar issues will be discussed in Sect. 5 .

\subsection{Analysis of the Multi-Point Relays}

The distinctive feature of OLSR, and one of the most debated, is the way MPRs are selected [16]. We introduce some 


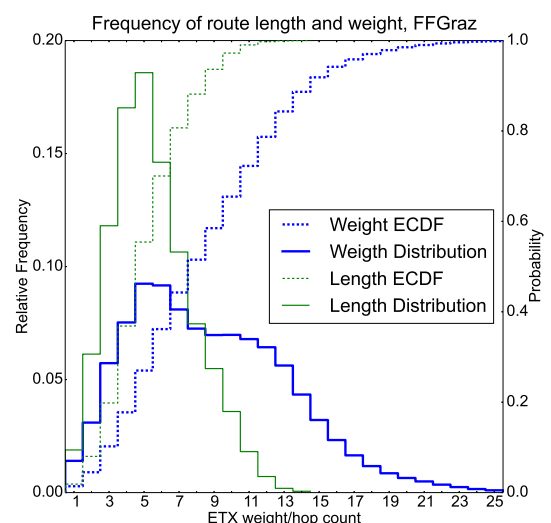

(a) Funk Feuer Graz

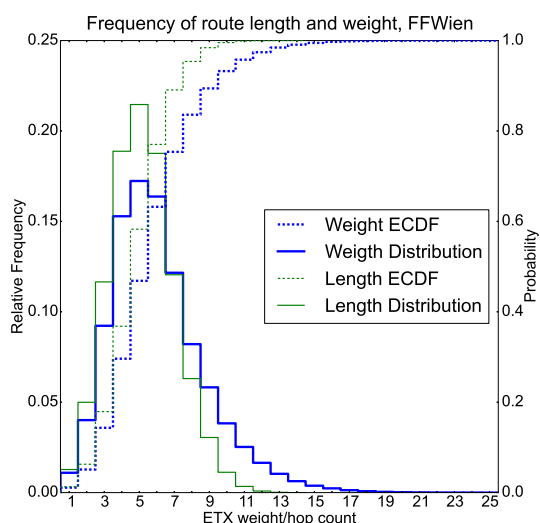

(b) Funk Feuer Wien

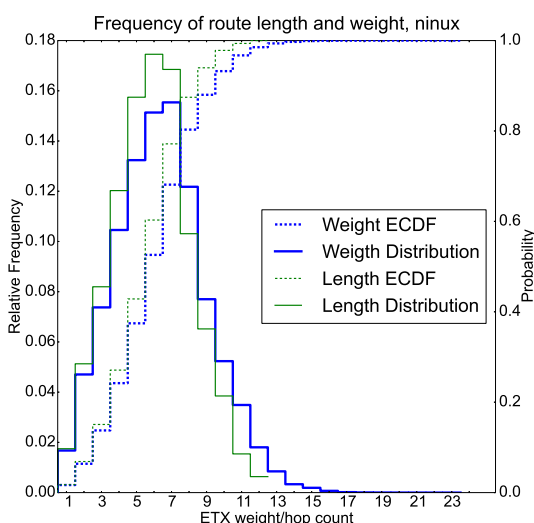

(c) Ninux

Figure 8: Empirical distribution (solid lines, left $y$ axis) and ECDF (dashed lines, right $y$ axis) $w\left(P_{i, j}\right)$ based on hop count (green/thin line) and ETX (blue/thick line)

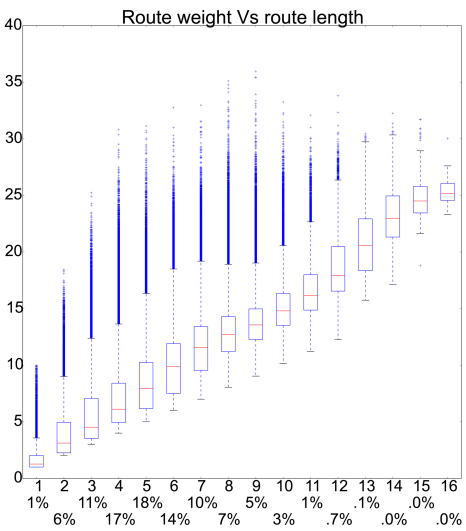

(a) Funk Feuer Graz

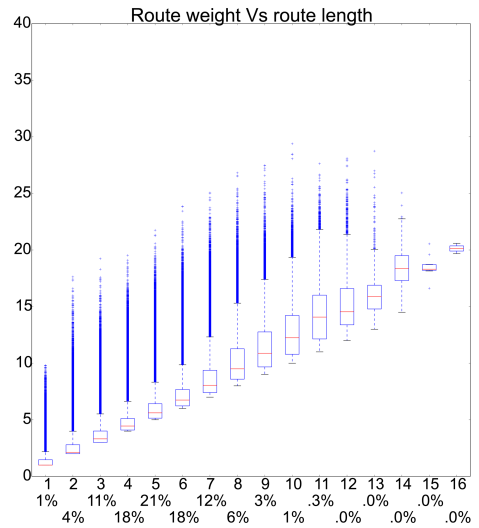

(b) Funk Feuer Wien

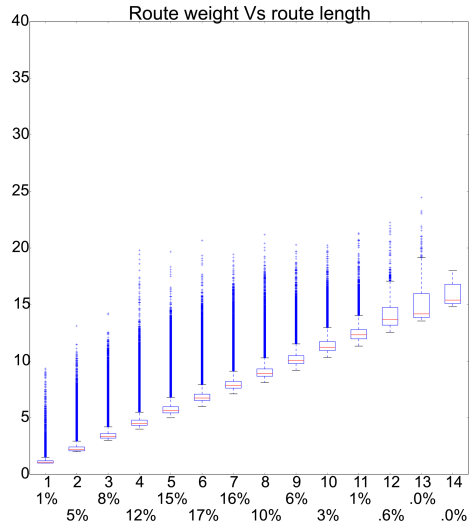

(c) Ninux

Figure 9: Boxplots of the distribution of $w\left(P_{i, j}\right)$ vs the number of hops in the path; boxes represent the 1 st and 3rd quartile, the whiskers are set to 1.5 times the Inter Quantile Range. X axis reports also the percentage of routes with the corresponding length

notation to further discuss this subject. Let $j$ be a node of the network, $N_{1}(j)$ is its neighbors' set and $M(j) \subseteq N_{1}(j)$ is the set of MPRs chosen by $j, M_{g}$ is the union of all $M(j) ; S_{g}=\left\|M_{g}\right\|$. $S(i)$ is the selector set of MPR $i$ and the symbol $j \rightarrow i$ means $j \in S(i)$.

As a general policy, $S_{g}$ should be small, since every MPR generates signalling that is propagated to all nodes. The heuristic proposed in the OLSR RFC tries to minimize each $M(i)$, and it has been shown that it produces results close to the local optimum in dense scenarios [20, 21]. Minimizing also the intersection of each $M(i)$ reduces $S_{g}$ even further [19]. Completely decoupling the choice of $M(i)$ from the links' quality may lead $i$ to choose an MPR $j$ even if the ETX on the link is high (bad link). This has two drawbacks. The first is the potential computation of sub-optimal paths. As each node has only an approximated view of the network based on HELLO and TC messages, an $M_{g}$ built ignoring quality metrics may hide (as we explained in Sect. 2.2) links with high quality, and lead to compute sub-optimal routes (we call this conjecture [A], as it is one of the assumptions upon which many decisions and choices are taken in tuning OLSR, but rarely verified). The second is that the robustness of the diffusion of signalling may be reduced. In fact, $i$ may not receive TCs from $j$ and thus have outdated information on the network topology (conjecture [B], also this one an assumption often made, but rarely verified).

The OLSRd community has initially implemented its own quality-aware MPR selection algorithm that chooses for each 2-hop neighbor the 1-hop neighbor that maximizes the quality on the two-hop path, an idea initially introduced in [22]. Consequently $M_{g}$ in a dense network constantly changes, since it doesn't depend on the links availability, that is assumed to be slowly changing, but on the link quality that can be much more variable. A fast changing $M_{g}$ can have two bad consequences. The first one is that also the routing may be changing frequently. When $P_{i, j}$ changes, even if $w\left(P_{i, j}\right)$ remains similar, other quality parameters of the route can vary (for instance, ETX doesn't estimate the capacity of a link). The protocols and the applications at the upper layers can be highly impacted by such variations. The second one is that every time there is a change in $M_{g}$ the network needs some time to propagate this information with 
HELLO and TC messages. When the information propagation time becomes comparable to the time between changes there is a high risk of network instability. The result of a large period of trial and error [23] was that the developers of OLSRd, as a default configuration, force every node to select all its neighbors as MPR, in practice renouncing to the optimization introduced by the MPRs. As said, this is also the approach used in the three networks under evaluation.

With the data-set available we can evaluate the impact of different choices of MPRs. The introduction of quality-aware MPRs selection raises many issues and gives many choices, which are often used arbitrarily. We want to study if conjectures [A] and [B] justify the use of quality metrics or not and how different MPRs selection influences the topology quality. We call $M_{g}^{q}$ the set of MPRs chosen with the link-quality maximization metric, and $S_{g}^{q}$ its size.

\subsection{Overhead reduction}

Since we have the full topology and all $c_{l}$ we can compute off-line $M_{g}$ and $M_{g}^{q}$ reproducing both metrics. Figure 11a and 11 b report $S_{g}$ and $S_{g}^{q}$ vs time (snapshots) for a one-day subset and show two relevant facts: i) $S_{g}^{q}$ largely exceed $S_{g}$; and ii) $S_{g}$ for FFGraz is smaller compared to NNX, but this is the opposite for $S_{g}^{q}$. The reason for $i i$ is that FFGraz is larger and denser NNX, so that the $S_{g}$ reduction achievable on FFGraz is higher (the MPR choice with the RFC heuristic is more efficient in dense networks). In Ninux the physical topology and the low density strongly influences the choice of $M_{g}$, and $S_{g}$ cannot be reduced.

To quantify how this impacts the signalling overhead, Fig. 11c reports $L_{t}$, which estimates the normalized load per link per generated TC:

$$
L_{t}=E_{l}\left[\rho_{t}^{l}\right] ; l \in \mathcal{L}
$$

where $E_{l}[\cdot]$ is the average over all links and $\rho_{t}^{l}$ is the total number of TCs transmitted on link $l$ in each TC interval. It is clear that reducing $S_{g}$ is fundamental to reduce the signalling overhead. FFGraz has an average of $10 \%$ more nodes than NNX, but also 35\% links more than NNX, this explains why FFGraz has less TC messages per link.

This estimation does not consider TC aggregation or fish-eye strategies, that can reduce the global amount of TC messages regardless of MPR selection strategy. Note also that Fig. 11c only reports TC messages, but MPRs are used to re-broadcast any other signalling messages, such as HNA messages, or messages needed to spread the association between a node and a list of its available services. Thus, it is of paramount importance to reduce the number of MPRs to keep the signalling bearable when the network size grows.

\subsection{Route quality}

Let's now analyze the impact of MPR selection on route calculation. The base of conjecture [A] is that a node $i$ receives topology information from HELLO and TC messages. Thus, it has only an approximated view of the network. We want to quantify how much this can negatively influences routing.

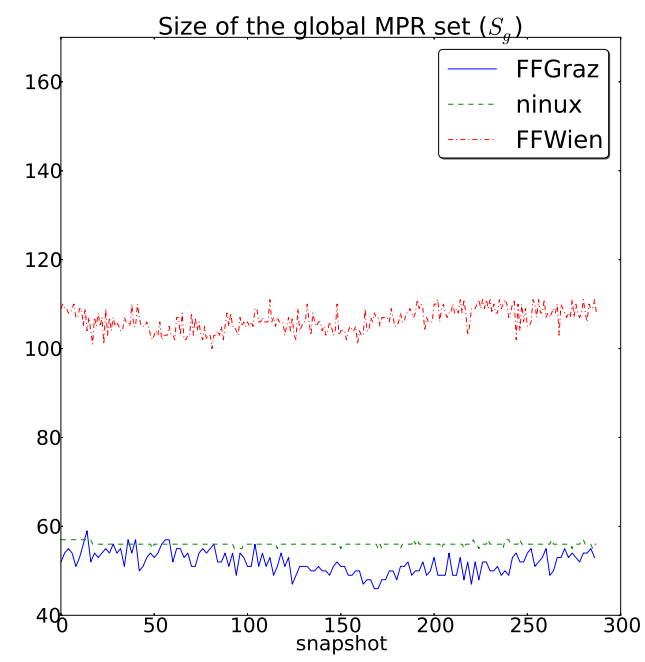

(a) Number of MPRs $\left(S_{g}\right)$

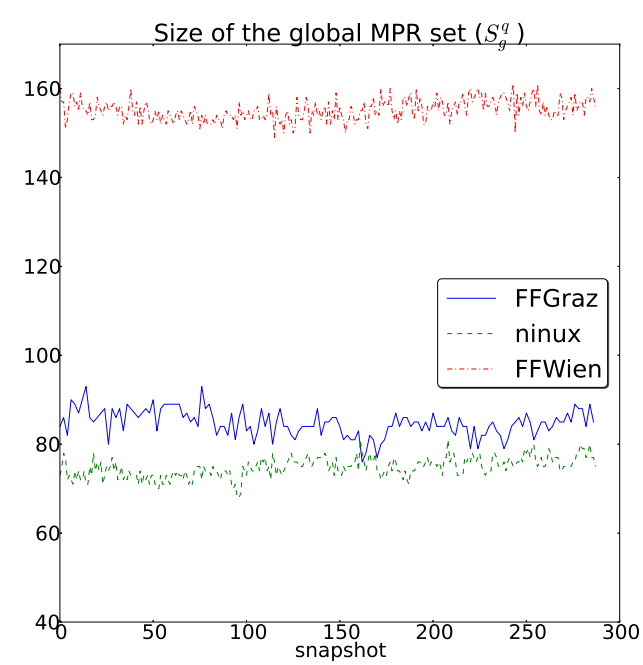

(b) Number of MPRs $\left(S_{g}^{q}\right)$

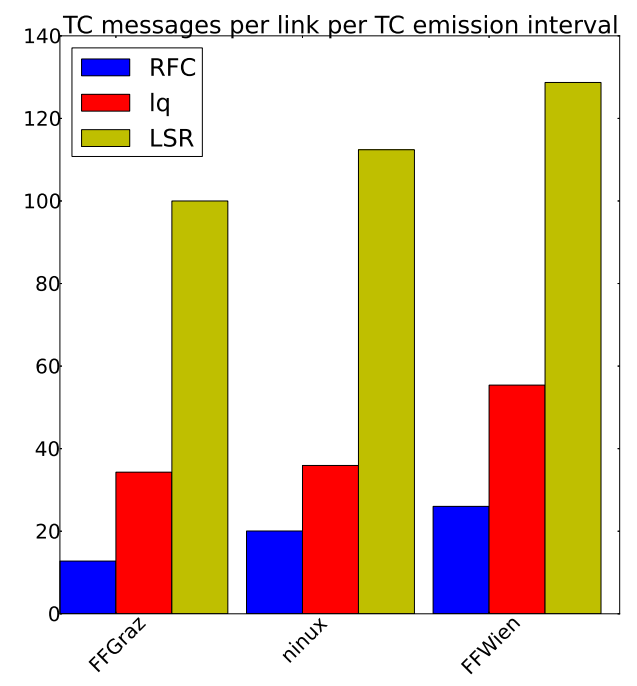

(c) Estimated TC load $L_{t}$ 
Define $G_{i}\left(\mathcal{N}, \mathcal{L}^{\prime}\right)$ as the approximated view of the network graph $G$ by node $i$. $G_{i}$ has the same number of nodes of the original graph $G$ but has a smaller number of edges. $\mathcal{L}^{\prime} \subseteq \mathcal{L}$ contains the edges $l_{k j}$ for which at least one of the following condition holds:

1. $k=i$, or $j=i$

2. $k \in N_{1}(i)$ or $j \in N_{1}(i)$

3. $k \in M_{g}$ and $j \rightarrow k$ or $j \in M_{g}$ and $k \rightarrow j$

In practice, each node $i$ knows only the links to its neighbors, the links between its 1-hop neighbors and its 2-hop neighbors and the link between any MPR in the network and its selectors. For this analysis the MPR are chosen using the heuristic from the RFC, without considering link qualities.

Given $G_{i}$ we can compute the routing table $R_{i}(j)$ for every node $i$, and hence, for every $(i, j)$ in $\mathcal{N} \times \mathcal{N}$ compute the effective path $P_{i j}^{\prime}=\left\{i=p_{0}, p_{1}=R_{p_{0}}(j), p_{2}=R_{p_{1}}(j) \ldots j\right\}$ that packets follows from $i$ to $j$. Comparing $w\left(P_{i j}^{\prime}\right)$ with $w\left(P_{i, j}\right)$ gives a measure of the real impact of conjecture [A].

Figs. 12a, 12b, and $12 \mathrm{c}$ report $w\left(P_{i j}^{\prime}\right)$ with $w\left(P_{i j}\right)$ for all pairs $(i, j)$ averaged on all the snapshots and ranked by their weight. Surprisingly enough, the difference is extremely limited, in $98 \%$ of the samples the same routes are chosen and the curves overlap. This can be explained observing that even if node $i$ doesn't have enough information to compute the globally best route, it has enough information to send the packet to the most convenient next-hop. Then, at any hop, the node that is routing the packet has a full knowledge the graph up to its 2-hop neighborhood, and can choose again the most convenient next hop.

This result is particularly relevant considering that OLSR2 [24], which is currently in its final stage of standardization distinguishes between MPRs used to broadcast signalling and MPRs used for routing. This choice was taken because of conjecture $[\mathrm{A}]$. At least for these three networks, and we believe for WCNs and meshes in general, this difference doesn't hold: maintaining two sets of MPRs may be an unjustified overhead.

OLSR is still one of the most used protocol in WCNs, even if the communities tried to mitigate its limitations creating new protocols, such as BATMAN, that is also widely used [23]. BATMAN, being a distance-vector protocol generates smaller signalling messages, but it doesn't give a full view of the network to each node. We believe that the data we gathered show that a correct implementation of OLSR can greatly reduce the overall signalling, while maintaining stability properties. We also believe that, even if this information is currently not used, the knowledge of the full network topology (or at least of an approximated one) can be beneficial to fine-tune the protocol properties. For instance, the frequency of the signalling could be raised for nodes that prove to be central in the topology, and lowered for nodes that are in the periphery. The next Section shows how to compute centrality metrics within OLSR, something that would be much more difficult to do with a distancevector routing protocol.

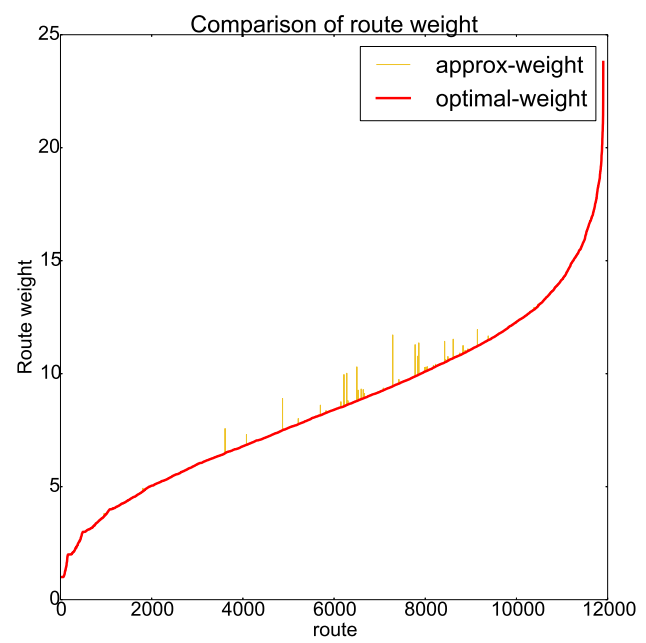

(a) Funk Feuer Graz

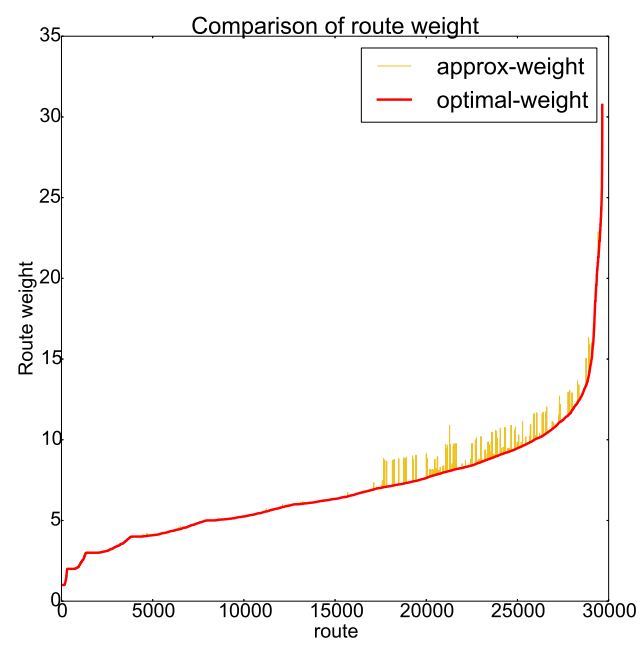

(b) Funk Feuer Wien

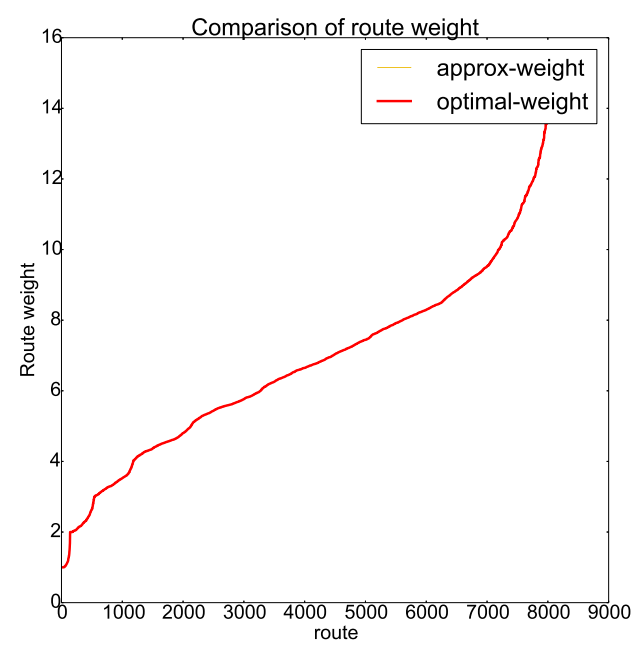

(c) Ninux

Figure 12: Route weights with and without the approximation due to MPRs 


\section{Centrality and Robustness Metrics}

In graph theory, centrality metrics have been largely used to identify the properties of nodes. In particular, in social science the centrality of a node is often used to determine the influence that a person has on the other participants of the social network. In the context of wireless networks, centrality has not received much attention up to recent times $[14,15]$.

The concept of centrality in a specific graph is not unique, in this paper two definitions of centrality are considered, shortest path group betweenness centrality $C_{s p}$, or simply betweenness, and group closeness centrality $C_{c}$ (definitions can be found in [25]). Computing group centrality is a hard task, so that heuristics must be used for networks of the size we are considering.

Robustness metrics are used, instead, to study the robustness of the network against nodes or link failures. We will quantify the robustness of the network graph, but also introduce an initial evaluation of how the introduction of MPRs can impact the diffusion of TC messages.

\subsection{Centrality Metrics}

The $C_{s p}(k)$ of node $k$ is defined as the fraction of shortest paths $P_{i, j} \forall i, j \in \mathcal{N}$ passing through $k$. If the traffic matrix is uniform or unknown, $C_{s p}(k)$ is an unbiased estimator of the fraction of traffic that a node will route. If one wants to place a traffic analyzer in the network (for instance an Intrusion Detection System (IDS)), the node with the highest $C_{s p}$ is the best choice to intercept the highest fraction of the overall traffic.

The closeness centrality $C_{c}(k)$ of node $k$, instead, is an estimation of the cost needed to spread an information from $k$ to all the nodes in the network. The definition that best serves the purposes of this paper is that $C_{c}(k)$ is the average distance from $k$ to any other node $i$ in the network ${ }^{5}$. If one wants to place a service in the network (a web server, a streaming server, etc.) the node with the lowest $C_{c}$ is the best choice to minimize its distance to any node in the network. For both centrality measures the weighted graph is used, so that distances are weighted using ETX; thus, $C_{c}(k)$ is the average number of wireless frames that will be needed to successfully send one IP packet from any node $i$ to the service placed on $k$ (or vice versa).

The definition of both metrics can be extended to groups of nodes. The group betweenness of a group $\gamma$ of nodes is defined as the fraction of $P_{i, j} \forall i, j \in \mathcal{N}$ passing through at least one node of $\gamma$. Again, if one wants to place an IDS on a group $\gamma$ of nodes in order to maximize the overall fraction of traffic analyzed, the group with the highest $C_{s p}(\gamma)$ is the best choice.

The closeness group centrality of $\gamma$ is the average of the minimum shortest path from any node $i$ to any of the nodes in $\gamma$. Among all the groups of the same size, the more central one is the one with the lowest $C_{c}(\gamma)$.

${ }^{5}$ Closeness centrality is generally defined as the inverse of $C_{c}(k)$. We prefer to use $C_{c}(k)$ since coupled with the ETX metric, its value has a direct correspondence with the average number of wireless frames sent along the path from any node $i$ to $k$.
Following the previous example, if a service can be replicated on a set $\gamma$ of nodes, then the group with lowest $C_{c}(\gamma)$ is the best choice. As a further example imagine that $C_{c}(\gamma)$ can be used as a metric to place Internet gateways. The group with the lowest $C_{c}(\gamma)$ is the group that will give the best average Internet connectivity to the nodes in the network. Since the ETX metric of a link is an estimation of the number of frames needed to successfully deliver an IP packet on that link, $C_{c}(\gamma)$ is directly proportional to the average load (in terms of number of frames) on the network generated by the traffic directed to the Internet. This is of particular importance also for a commercial Wireless ISP that needs to design a mesh network to deliver internet connectivity. The number and the position of the gateways in the network must be decided in order to distribute the load on the whole topology and reduce the chances of creating bottlenecks.

\subsubsection{Computing Group Centrality}

Finding the group $\gamma$ with the highest group betweenness has been shown to be an NP hard problem while a brief analysis of the literature did not produce any complexity estimation for the closeness group centrality as we defined it.

The size of the networks under consideration does not allow the use of an exhaustive search on all the groups of size $k$ to find the global optimum, thus we implemented a greedy algorithm we call GR1 to estimate the best group. At each step $h$, given the solution computed at the previous step $\gamma_{h-1}$, for every $i$ in $\mathcal{N}$, GR1 composes a new set $\gamma_{h}^{i}=\gamma_{h-1} \cup\{i\}$ and computes $\mu_{i}=C_{s p}\left(\gamma_{h}^{i}\right)-C_{s p}\left(\gamma_{h-1}\right)$. It ranks all the $\gamma_{h}^{i}$ for the corresponding $\mu_{i}$ and selects the one with highest $\mu_{i}$. The difference from the optimal $C_{s p}\left(\gamma_{o}\right)$ and $C_{s p}\left(\gamma_{k}\right)$ produced by GR1 has been shown to be lower bounded by a constant in the general case [26], moreover GR1 performs particularly well on scale free networks [27]. Some of our networks show a scale free behaviour, so we chose this greedy algorithm to compute the best centrality measures. Nevertheless, since our betweenness centrality measure is slightly different from the one used in [26] and closeness centrality was not considered in that work, we validated the greedy algorithm with a GRASP (Greedy Randomized Adaptive Search Procedure) approach, and in particular, with a parallel multiple-walk independent-thread procedure [28] that we called GR2. GR2 works as GR1, but instead of always choosing the $\gamma_{h}^{i}$ with highest $\mu_{i}$ it selects a restricted candidate list of the first 5 and randomly picks one of them, with probability proportional to $\mu_{i}$. We ran GR2 with 16 independent parallel processes for each network graph and chose the best solution. If the problem we want to solve has many local optimum values far from the global one, GR2 should perform substantially better GR1. Instead we verified that GR2 produces a negligible improvement in both centrality metrics, less than $1 \%$ on average compared to GR1. This confirms that on the topologies of our data-set GR 1 produces solutions very close to the optimum.

Fig. 13a shows the group betweenness centrality of groups up to size 5. It is clear that a motivated attacker, controlling a few nodes is able to sniff a very large portion of the traffic. In light of the recent Datagate privacy scandal, WCNs have attracted the attention of mainstream media as a bottom-up alter- 


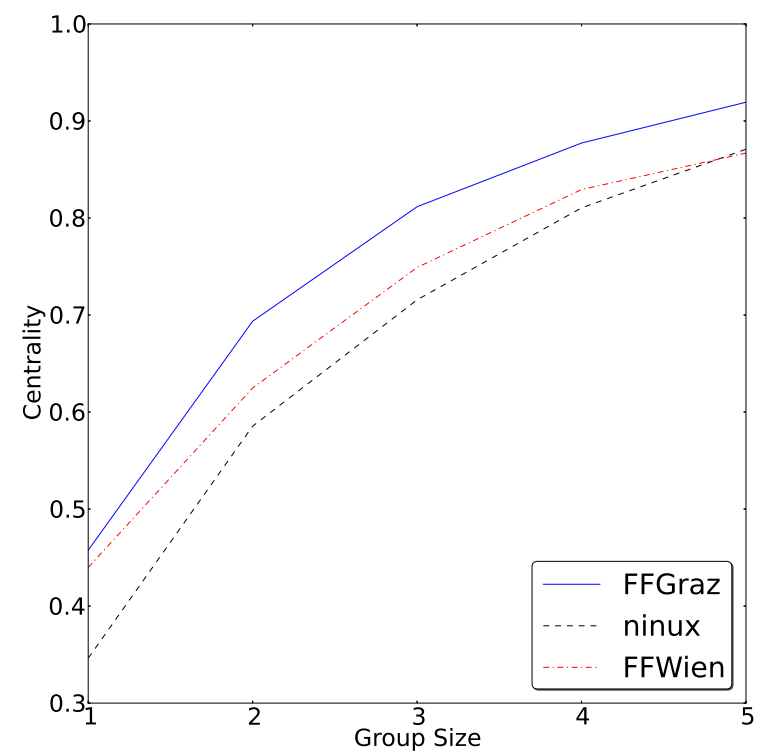

(a) Group betweenness centrality

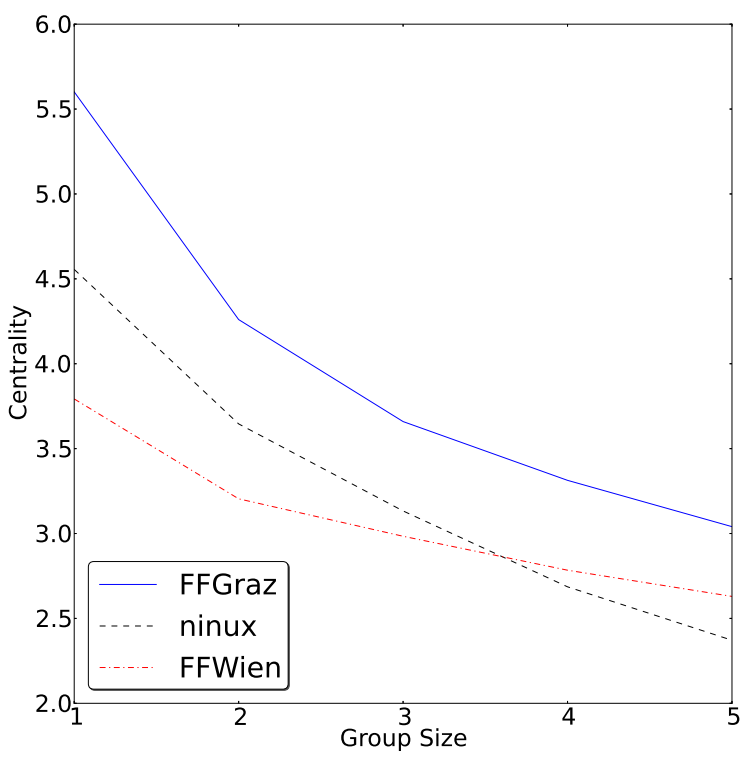

(b) Group closeness centrality

Figure 13: Group Betweenness and closeness Centrality of the best groups with size from 1 to 5

native to avoid privacy infringement ${ }^{6}$. Our analysis on real networks shows instead that using a peer-to-peer technology does not guarantee by itself to have networks that are robust to interception.

\footnotetext{
${ }^{6}$ See the New York Times, "Home Wireless Network Keeps the Snoops Away"

http://www.nytimes.com/2013/11/14/technology/personaltech/ homemade-wireless-networks-keep-the-snoops-away.html?smid= $\mathrm{pl}$-share\&_r $=0$
}

The other side of the coin is that a distributed monitoring system placed on a small subset of nodes will be able to intercept and analyze the majority of the traffic of the network, and also to counter external attacks to privacy.

Fig. 13b instead shows that with a careful choice of $\gamma$ the average network load generated by traffic directed to the gateways or to a set of servers can be cut down significantly. Recall that with OLSR, each node exposes the private networks it is connected to using specific messages (HNA messages). By extension, it can also export the offered services (as in [29]). OLSR thus offers an easy and efficient way (since it exploits the MPR system) to spread the knowledge that a particular service is present in a set $\gamma$ of nodes. Every other node is thus able to use the service on the host in $\gamma$ that is more convenient to reach.

\subsection{Robustness}

The robustness of a graph is an estimation of the impact of failures on the graph connectivity, and can be measured with metrics introduced in [30] and based on percolation theory. Given a connected graph $G(\mathcal{N}, \mathcal{L})$ we remove a set $F$ made of $f$ random edges and we call $G^{\prime}\left(\mathcal{N}^{\prime}, \mathcal{L}^{\prime}\right)$ the main connected component of the resulting graph. When an edge is removed the connectivity of the graph may change, meaning that some nodes can be isolated. The robustness of $G(\mathcal{N}, \mathcal{L})$ is the ratio $\frac{\left\|\mathcal{N}^{\prime}\right\|}{\|\mathcal{N}\|}$, averaged over a sufficient number of random choices of $F$. The rationale behind this metric is that if the robustness stays close to 1 , the network is still functionally behaving as a network when subject to $f$ uncorrelated failures. Instead, if it drops to values close to 0 , the network is fragmented into a multitude of isolated networks. In practice, there is no network anymore. The same measure is repeated for growing values of $f$.

We compute the robustness of each snapshot with 30 random choices of $F$ and growing value for $f$. Instead of expressing the robustness against the absolute number of failures, it is convenient to express it against relative number of failed links, so that different networks can be compared. Since the number of edges in each snapshot (even of the same network) lightly varies, we quantize the relative number of failures to a percentage: $k=\lceil 100 * f /\|\mathcal{L}\|\rceil$. Then for each network we call $r(k)$ the robustness averaged over every snapshot.

Since ETX expresses the reliability of a link, instead of choosing the edges with an uniform distribution the probability of being removed is proportional to the normalized value of ETX.

We choose to compute the robustness in four different ways:

1. On the full graphs;

2. On the full graphs, but choosing the links to purge only within the core network, i.e., only the links that connect non-leaf nodes;

3. On the MPR sub-graph $G_{m}\left(\mathcal{N}, \mathcal{L}_{m}\right)$ in which a link exists only from a selector to its MPR. MPRs are chosen with the RFC heuristic;

4. On the MPR graph $G_{m}^{q}\left(\mathcal{N}, \mathcal{L}_{q}\right)$ built as the previous one but with MPRs chosen based on link quality. 
The last two ways affect the signalling network only. To better explain this we refer to $G_{m}$ but the considerations are the same for $G_{m}^{q}$. When a node $m$ that is not an MPR generates an HELLO packet to show its presence, this information is distributed to the whole network by at least one MPR $i$ for which $m \rightarrow i$. Similarly, when a TC message is produced by $i$, the information it carries moves from $i$ to any other MPR $j$ for which $i \rightarrow j$, and this is repeated at every hop. If one link is broken, TCs can still arrive to every destination using alternative paths. But, if one or multiple failures make $G_{m}$ disconnected, the network is logically broken. If this condition is not transitory, the routing needs to be reconfigured and temporarily some routes will be missing. Computing $r(k)$ on $G_{m}$, we give an estimation of how robust is the delivery of routing information on $G_{m}$ that directly depends on $S_{g}$ and $S_{g}^{q}$.

Fig. 14a shows the robustness of the network and of the network core. First of all we can observe that all the networks are quite robust. If we remove $10 \%$ of links, that is a relevant portion of the links, robustness is higher than 0.9. Second, robustness is higher for networks with higher average degree, and this is particularly evident between FFGraz and NNX. Even if their size is similar, in NNX, the choice of minimizing the number of links per device increasese the average quality per link, but decreases the robustness.

There is a visible difference between the robustness of the full graph and of the core graph. In all three cases, for low values of $k$ the core network is more robust that the full network, then the two curves intersect. To understand this, consider that if we remove a random link, if this link connects a leaf node $\left\|\mathcal{N}^{\prime}\right\|$ decreases by one unit. Instead, if the core network is well connected, removing one link does not necessarily decrease $\left\|\mathcal{N}^{\prime}\right\|$. Since FFGraz and FFWien have a higher degree than NNX the intersection happens for higher values of links removed.

Fig. $14 \mathrm{~b}$ presents the robustness of the MPR sub-graphs. It is intuitive that $G_{m}$ will be less robust than $G_{m}^{q}$ since it has a smaller $M_{g}$. For this reason we normalize the number of failed links to the number of MPRs. Thus, we plot $r\left(k^{\prime}\right)$ where $k^{\prime}=\left\lceil 100 \times f / S_{g}\right\rceil$, and $r\left(k^{\prime \prime}\right)$ where $k^{\prime \prime}=\left\lceil 100 \times f / S_{g}^{q}\right\rceil$ instead of $r(k)$ respectively. This metrics express the robustness normalized to the respective sizes $S_{g}^{q}$ and $S_{g}$, notice that $k^{\prime}$ and $k^{\prime \prime}$ can be larger than 100 , so that they are not normalized parameters strictly speaking, as they are referred to a number of nodes and not to a number of links. As a result we see that the curve referring to quality-based MPR selection (solid lines, marked with "lq" in the figure legend) stays below the curve referring to the standard RFC implementation. This is relevant with respect to conjecture $[\mathrm{B}]$ : we can finally say that using the RFC heuristic produces a small reduction in terms of robustness, which is largely compensated, from a global management point of view, by the reduction of MPRs, and consequently of signalling, making quality-based MPR selection questionable.

Similarly, robustness can be evaluated against the failure of nodes, simply considering a random set of $f$ nodes, normalized on the size of $\mathcal{N}$. Fig. 15a and Fig. 15b show the robustness of the network graph to the failure of nodes. We evaluate the robustness against random failures and targeted attacks. In the first case, the nodes are randomly removed from the networks,

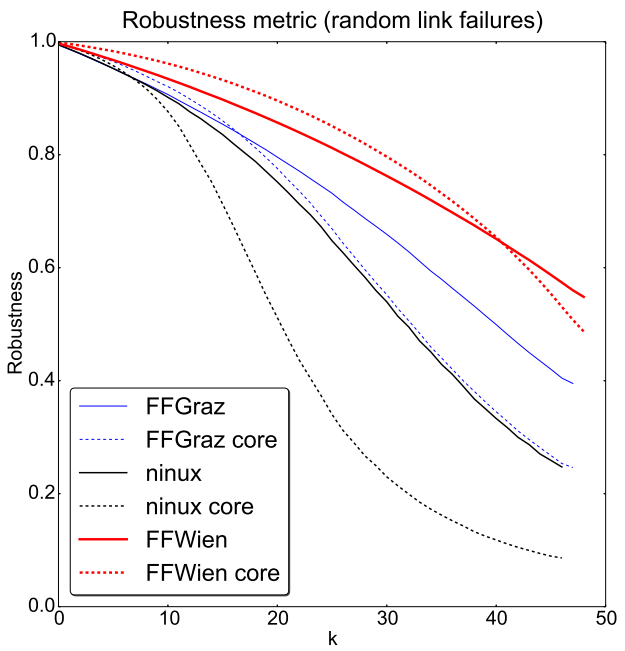

(a) Robutness computed on the network graph

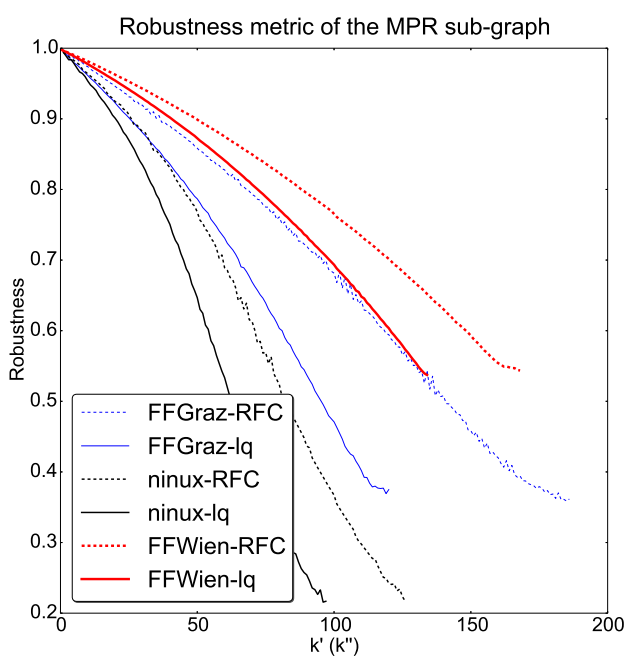

(b) Robutness computed on the MPR sub-graph

Figure 14: Robustness metric for link failures on the network graphs and on the MPR sub-graphs with both RFC and ETX based heuristics

in the second, we start removing nodes from the one that have the highest degree. This second case resembles the actions of a rational attacker that wants to provoke the highest damage to the network.

The graphs show that due to the high skewness of the degree distribution, the networks are quite resistant to random failures, but are pretty weak against targeted attacks. 


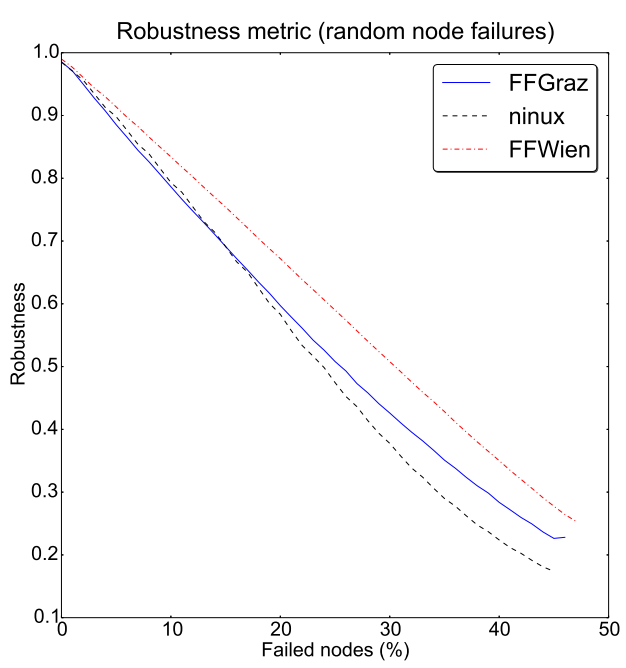

(a) Robustness to random node failures

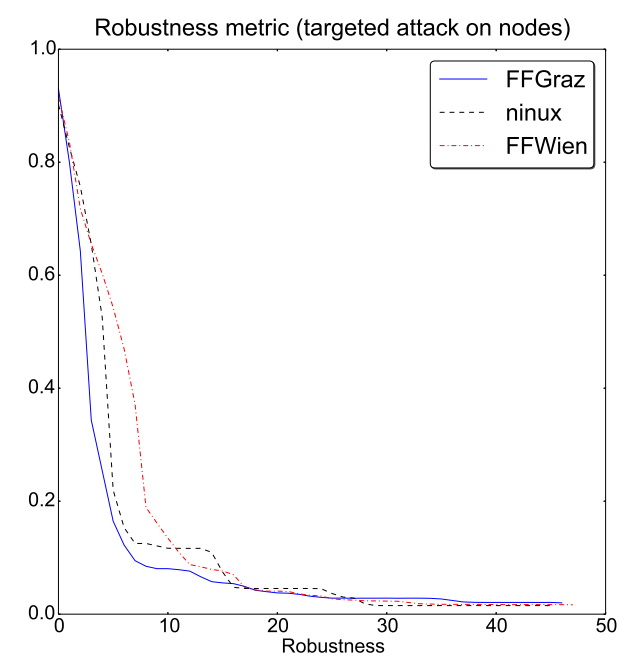

(b) Robustness to targeted attacks

Figure 15: Robustness metric for node failures

\section{Conclusions}

Wireless Community Networks represent a thriving mix of technical solutions and social participation. Their open nature allows users and researchers to participate, experiment and collect information on how the networks work. In this paper we used the data collected on three large WCNs to study their main topology features, the centrality of their nodes and their robustness. We also used the data-set to investigate on the role of MPRs in an OLSR-based network, something that, to the best of our knowledge, has never been done on such a large scale. The results show that, even with some differences, WCNs are pretty stable networks, the average quality of the links is good, and thus, the weights on the routes are close to their length in terms of hops. We also showed that the use of MPRs can reduce the signalling, without a strong impact on the quality of the routes.
WCNs are often perceived as a tool that users can build to escape control and protect their privacy. We showed that the structural properties of the networks alone do not make them more robust against coordinated attacks, since the traffic is concentrated on a few important nodes (as we showed using centrality metrics) and the connectivity relies on a few important nodes (as we showed using robustness metrics).

A preliminary version of the data-set and of the code used for the analysis is already available for public use and the possible falsification of our results. We are actively documenting the code used and improving its usability and the access to the database as full Open Data ${ }^{7}$.

Many further research topics can be explored on the data-set, for instance, we plan to study the properties of the most central nodes, verify how much they change with time and how we can use heuristics to avoid the complex algorithms we have used in our analysis. Central nodes may behave differently (for instance, their signalling can be more frequent) and the routing protocol could take advantage of the knowledge of the full topology, contrarily to what happens now.

\section{Acknowledgemts}

We greatly thank all the people from the community networks that contributed to the gathering of the data. In particular, Aaron Kaplan and Ralf Schlatterbeck for the Funk Feuer networks and Claudio Pisa for the Ninux network.

\section{Bibliography}

[1] S. Jain, D. Agrawal, Wireless community networks, IEEE Computer $36(8)$.

[2] P. Frangoudis, G. Polyzos, V. Kemerlis, Wireless community networks: an alternative approach for nomadic broadband network access, IEEE Communications Magazine 49 (5).

[3] A. Neumann, I. Vilata, X. León, P. E. Garcia, L. Navarro, E. López, Community-lab: Architecture of a community networking testbed for the future internet, in: IEEE International Conference on Wireless and Mobile Computing, Networking and Communications (WiMob), Barcelona, Spain, 2012.

[4] L. Maccari, An analysis of the ninux wireless community network, in: IEEE International Conference on Wireless and Mobile Computing, Networking and Communications (WiMob), Lyon, France, 2013.

[5] L. Maccari, R. Lo Cigno, Waterwall: a cooperative, distributed firewall for wireless mesh networks, EURASIP Journal on Wireless Communications and Networking 2013 (1).

[6] L. Maccari, R. Lo Cigno, Betweenness estimation in OLSR-based multihop networks for distributed filtering, Journal of Computer and System Sciences 2014 (3).

[7] R. Draves, J. Padhye, B. Zill, Comparison of routing metrics for static multi-hop wireless networks, in: ACM Conference on Applications, Technologies, Architectures, and Protocols for Computer Communications, (SIGCOMM) Portland, USA, 2004.

[8] J. Bicket, D. Aguayo, S. Biswas, R. Morris, Architecture and evaluation of an unplanned 802.11B mesh network, in: ACM International Conference on Mobile Computing and Networking (MOBICOM), Cologne, Germany, 2005.

[9] V. Brik, S. Rayanchu, S. Saha, S. Sen, V. Shrivastava, S. Banerjee, A measurement study of a commercial-grade urban wifi mesh, in: ACM Conference on Internet Measurement, Vouliagmeni, Greece, 2008.

\footnotetext{
${ }^{7}$ All the code and data are available at http://disi.unitn.it/maccari/CN/
} 
[10] K. LaCurts, H. Balakrishnan, Measurement and analysis of real-world 802.11 mesh networks, in: ACM Conference on Internet Measurement, Melbourne, Australia, 2010.

[11] M. Afanasyev, T. Chen, G. Voelker, A. Snoeren, Usage patterns in an urban WiFi network, IEEE/ACM Transactions on Networking 18 (5).

[12] B. Braem, C. Blondia, C. Barz, H. Rogge, F. Freitag, L. Navarro, J. Bonicioli, S. Papathanasiou, P. Escrich, R. Baig Vias, A. L. Kaplan, A. Neumann, I. Vilata i Balaguer, B. Tatum, M. Matson, A case for research with and on community networks, SIGCOMM Comput. Commun. Rev. 43 (3).

[13] D. Vega, L. Cerda-Alabern, L. Navarro, R. Meseguer, Topology patterns of a community network: Guifi.net, in: IEEE International Conference on Wireless and Mobile Computing, Networking and Communications WiMob, Barcelona, Spain, 2012.

[14] D. Katsaros, N. Dimokas, L. Tassiulas, Social network analysis concepts in the design of wireless ad hoc network protocols, IEEE Network 24 (6)

[15] M. Kas, S. Appala, C. Wang, K. Carley, L. Carley, O. Tonguz, What if wireless routers were social? approaching wireless mesh networks from a social networks perspective, IEEE Wireless Communications 19 (6).

[16] O. Liang, Y. A. Sekercioglu, N. Mani, A survey of multipoint relay based broadcast schemes in wireless ad hoc networks, IEEE Communications Surveys \& Tutorials 8 (4).

[17] T. Kitasuka, S. Tagashira, Density of multipoint relays in dense wireless multi-hop networks, in: IEEE International Conference on Networking and Computing (ICNC), Osaka, Japan, 2011.

[18] J. H. Ahn, T.-J. Lee, Multipoint relay selection for robust broadcast in ad hoc networks, Ad Hoc Networks 17 (0).

[19] L. Maccari, R. Lo Cigno, How to Reduce and Stabilize MPR sets in OLSR networks, in: IEEE International Conference on Wireless and Mobile Computing, Networking and Communications (WiMob), Barcelona, Spain, 2012.

[20] T. Clausen, P. Jaquet, Optimized Link State Routing Protocol (OLSR), RFC 3626 (oct 2003)

[21] B. Mans, N. Shrestha, Performance evaluation of approximation algorithms for multipoint relay selection, in: Mediterranean Ad Hoc Networking Workshop, Bodrum, Turkey, 2004.

[22] Y. Ge, T. Kunz, L. Lamont, Quality of service routing in ad-hoc networks using OLSR, in: Hawaii International Conference on System Sciences, Hawaii, USA, 2003.

[23] The OLSR story, from the developers of olsrd and batman.

URL http://www.open-mesh.org/projects/open-mesh/wiki/ The-olsr-story

[24] U. Herberg, T. Clausen, P. Jacquet, C. Dearlove, The optimized link state routing protocol version 2 , RFC.

URL draft-ietf-manet-olsrv2-19

[25] M. Newman, Networks: an introduction, OUP Oxford, 2009.

[26] S. Dolev, Y. Elovici, R. Puzis, P. Zilberman, Incremental deployment of network monitors based on group betweenness centrality, Information Processing Letters 109 (20).

[27] R. Puzis, Y. Elovici, S. Dolev, Finding the most prominent group in complex networks, AI communications 20 (4)

[28] M. G. Resende, C. C. Ribeiro, Parallel greedy randomized adaptive search procedures, Parallel Metaheuristics: A new class of algorithms 47.

[29] F. S. Proto, C. Pisa, The olsr mdns extension for service discovery, in: IEEE Communications Society Conference on Sensor, Mesh and Ad Hoc Communications and Networks. Rome, Italy, 2009.

[30] R. Albert, H. Jeong, A.-L. Barabsi, Error and attack tolerance of complex networks, Nature 406 (6794).

\section{Appendix A. Data Collection Details}

The OLSR topology can be biased by the choice of using multiple devices per super-node. We call a device an embedded system that includes at least a wireless and a wired interface. A super-node is composed of multiple devices connected to the same switch via the Ethernet interface.

Two example configurations of a super-node deserve to be described, in order to better understand the data collection

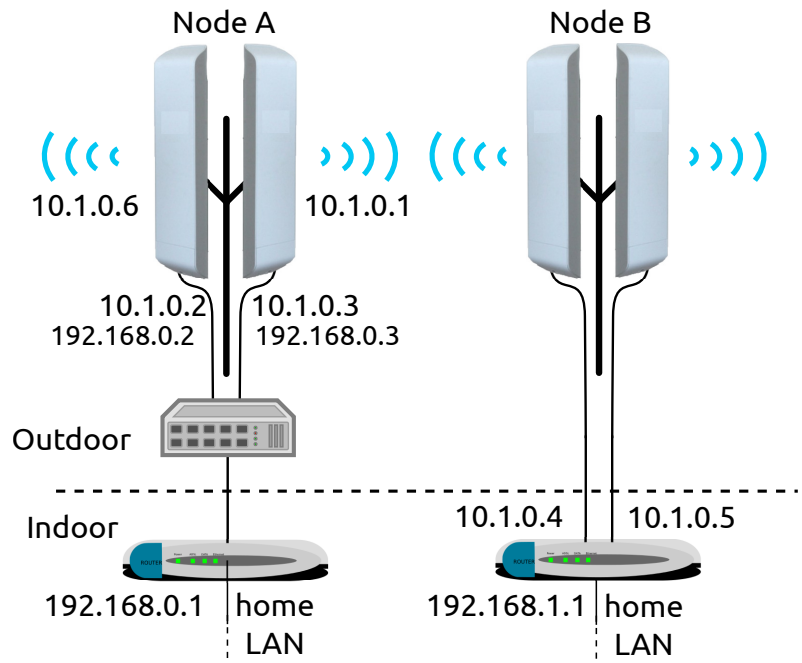

Figure A.16: Two example super-node configurations, each super-node is made of two devices.

phase. WCNs generally use two different addressing schemes for the mesh network and for the home LANs inside each user's house. In Fig. A.16 we used 10.X.X.X/8 for the mesh network and the classes 192.168.X.X/24 for the home LANs. In the simplest case, each wireless and wired interface of a device in a super-node is assigned an IP address in the class 10.X.X.X, and wired interfaces also have an IP address in the 192.168.X.X class. Each device runs its own instance of the OLSR routing protocol that manages both devices, making it practically an independent node. The 192.168.X.X addresses are treated from OLSR as private networks, that is, the OLSR protocol is not running on those networks, but OLSR generates special signalling packets to let each subnet reach the others. The wired interfaces are connected to a switch (normally placed directly in the rooftop) that is also connected to a device in the home of the user. This makes it easy to extend a super-node adding further devices since no change needs to be done on the existent ones. This configuration is used in node A in Fig. A.16.

Another configuration for super-nodes is to cable all the devices to the same router. The devices are configured as standard 802.11 APs/clients and do not run OLSR. The traffic coming from each device is isolated in the router using separated VLANs terminated on virtual interfaces on the router which runs OLSR. This entirely masks the super-node structure from the network. This configuration, at the cost of increased configuration complexity, has two advantages: $i$ ) signalling is reduced since only the router runs OLSR, and ii) the original firmware can be left on the devices as long as it supports bridging and VLAN tagging. This configuration is used in Node B in Fig. A.16.

From a routing point of view, there is difference in the two configurations; in the first case, the super-node corresponds to two different IP addresses in the network, with a wired link connecting them (thus, ETX will be fixed to 1). The resulting graph $G(\mathcal{N}, \mathcal{L})$ includes a clique of nodes with distinct IP addresses and edge weights constantly set to 1 . In the second, each super- 
node corresponds to only one host (with two different IPs).

We decided to merge super-nodes in one single logical node as this is functional to our analysis and also more representative of the network itself, for several reasons:

- The devices of a super-node all belong to the same person. If he is an attacker he will intercept traffic from all of them;

- They are powered via the same source, connected to the same switch and they share the physical installation, so that failures due to physical damage, power outage or human errors are correlated;

- Since the first configuration (Node A in Fig. A.16) of super-nodes is extremely inefficient, there are ongoing efforts to gradually migrate to the configuration with an external router (Node B in Fig. A.16)

The process of merging super-node's devices can not be applied using only data from the OLSRd daemon. Further information must be gathered from distinct sources, and this process was aided by the participants to the communities. For the FFGraz network the naming style of each device is a domainlike convention in the form "phisical-interface.device.node". This means that wifi0.device $0 . n o d e 0$ and wifil.device 0 .node 0 are the first and second wireless interfaces of the first device of the same node. Every 10 minutes FFGraz publishes the topology using this naming convention. We are thus able to group the interfaces and the devices corresponding to the same node. FFWien instead exposes a JSON interface to the internal node database. The presence of a centralized node database is common to many WCNs. It is used to monitor the evolution of the topology and to publish the network map on the community web server. Using the JSON interface we were able to map each IP address to each node, and compact the devices on the same node. For the FFWien network the data gathering process is split in two phases, one is the collection (every 5 minutes) of the OLSRd topology, and the second, once a day, is the access to the JSON interface to do node merging. The Ninux network currently does not expose a JSON interface but we had direct access to the node database updated every 5 minutes. Directly from the database we can access the topology, merge the nodes and collect the ETX values for each link.

Currently, we are not able to access information on the position of nodes for all the networks. The interested reader can find maps of the physical topology on the website of the three networks ${ }^{8}$.

\footnotetext{
${ }^{8}$ See http://map.ninux.org/ https://karte.ffgraz.net/ https://map.funkfeuer.at/wien/
} 